\title{
Phosphatidylinositol 4-Kinase Enzymes: Functional Roles and Potential for Drug Target
}

\author{
Manoj G Tyagi*, Deepasree Sukumaran, Saibal Das, Vishakha Tyagi and \\ Pulastya A Vora \\ Department of Pharmacology* and Department of Biotechnology+ \\ Rama Medical College, Hapur, Uttar pradesh \& Vellore Institute of Technology, Vellore, Tamilnadu, India
}

\begin{abstract}
The two types of phosphatidylinositol 4-kinases (PI-4Ks) synthesize phosphatidylinositol 4-phosphate (PI-4P), a member of the phosphoinositide family. Phosphoinositides (PIPs) are synthesized from phosphatidylinositol (PI), a lipid containing the myo-inositol head group. PI can be phosphorylated at positions 3,4 , and 5 of the inositol ring which allows for seven different PIPs. Indeed, all of these enzymes have been identified in the cell. For instance, one prominent function of PIPs is to serve as membrane markers typically in concert with organelle specific proteins. PI $(4,5) P_{2}$ is the main lipid determinant of the plasma membrane and PI3P and PI(3,5)P of the early and late endosomes. PI-4P is the main lipid determinant of the Golgi and trans-Golgi network (TGN) but, additionally, helps to define the specific character of the plasma membrane. This article reviews the recent developments in research on these enzymes and their potential for drug target.
\end{abstract}

\section{Introduction}

The Organelle-specific phosphoinositide distributions are maintained by the tight regulation of PI-kinases and PI-phosphatases. Four distinct PI-4kinases have been described in mammalian cells, including type II (PI4KII $\alpha$ and PI4KII $\beta$ ) and type III (PI-4KIII $\alpha$ and PI-4KIII $\beta$ ) kinases [1-3].The type II PI-4kinases are palmitoylated and thus are strongly associated with the membrane, particularly in the trans-Golgi apparatus, and to a smaller extent, in endosomes [4-5]. PI-4KIII $\beta$ localizes primarily to the Golgi apparatus, coincident with Arf1, a small GTP-binding protein. Although the molecular process of how these enzymes are linked to Golgi-derived biosynthetic transport remain unknown, they have all been implicated in Golgi function and secretion. Deletion of the gene for PI-4KIII $\alpha$ is embryonically lethal in mice, and its normal subcellular distribution is complex, with evidence for cytosolic, plasma membrane, and ER concentration. Recently, PI-4KIII $\alpha$ has also been identified as a critical host factor for hepatitis C viral replication [6-7]. With regards to biosynthetic trafficking, the phospholipid PI-4P localized to the Golgi apparatus has been implicated in the delivery of cargo from the Golgi to the plasma membrane (PM). The membrane phospholipid PI-4P in vivo is primarily produced by the action of PI-4Ks, although it can be also produced by dephosphorylating a higher PIP. All eukaryotes have two families of PI4Ks conserved from yeast to man called type II and type III PI-4Ks [8-9] that have different domain organization. The missing type I PI-4Ks is a historical artifact as it was later found out that the type I PI-4Ks were actually PI3Kinases. Type III kinases are known as typical PI-4Ks due to their similarity to PI3Ks and type II are known as atypical due to their dissimilarity to any other lipid kinases. Phosphatidylinositol phosphates (PIPs) are universal markers of intracellular membranes. Phosphatidylinositol 4-phosphate (PI-4P) is the single most abundant mono phosphoinositide that defines the membranes of the Golgi and TGN. It has been reported to play a key role in membrane biogenesis, vesicular transport, lipid dynamics, and protein and lipid sorting in the TGN. PI-4P is synthesized by phosphatidylinositol kinases (PI-4Ks) [10]. Humans have two type II PI-4Ks (alpha and beta) and two type III PI-4Ks (alpha and beta). On the other hand, the type III PI4Ks are carried by positive strand RNA (+RNA) viruses such as the Hepatitis C virus, poliovirus or SARS to create so-called membranous web, an extensively phosphorylated and modified membrane system dedicated to their replication [11].However, PI-4P produced by type II PI4Ks is used by intracellular bacteria (e.g. Legionella pneumophila, Chlamydia trachomatison). Thus, PI4Ks are a potential pharmacological target.Recent work has also established that different PI 4-kinase isoform permutations are required during receptor-activated PLC signalling and in Golgi-endosomal trafficking and all of which suggests that there is still much still to be discovered about this, the least well studied mammalian PI kinase family.

\section{Phosphatidylinositol 4 kinase and cancer biology:}

Uptil now increased expression of PI-4KII $\alpha$ and PI-4KII $\beta$ has been reported for a range of specific cancers with increased PI4KII $\alpha$ levels associated with augmented activation of the HER2 receptor kinase pathway, HIF production and angiogenesis [12]. In a interesting study, a potential anti-metastatic role for PI-4KII $\beta$ has emerged which involves this isozyme promoting the trafficking of CD81 tetraspanin proteins away from the plasma membrane into a population of intracellular trafficking vesicles that also contain actinin thereby 
inducing anti-migratory remodelling of the actin cytoskeleton [13]. Meanwhile PI-4KIII has been identified in non-biased screens as contributing towards a more aggressive metastatic phenotype of pancreatic ductal carcinoma cells and was identified as one of several proteins that mediate resistance to the chemotherapeutic agents gemcitabine [14-15] and cisplatin. However, unlike the well established case of constitutively active phosphoinositide 3-kinase mutations as found in PI-3KCA or phosphatase PTEN deletions which can both elevate $\mathrm{PI}(3,4,5) \mathrm{P}_{3}$ levels and drive oncogenic signaling and [16], it is significant that neither activating mutations of the PI 4-kinases nor deletions of PI4P phosphatases have yet been discovered in cancer.

\section{Estimation of PI4Kinase activity:}

The activity of PI4KII $\alpha$ can be determined by measuring ADP generation using an ADP-Glo kinase kit (Promega, USA) as previously described [17]. Briefly, $1 \mu \mathrm{g}$ protein in a kinase buffer containing $20 \mathrm{mM}$ Tris, pH 7.5, $150 \mathrm{mM} \mathrm{NaCl}, 0.2 \%$ Triton X-100 (or $0.1 \%$ Anzergent), $1 \mathrm{mM}$ EDTA and $20 \mathrm{mM} \mathrm{MgCl} 2$ was used in this assay. PI dissolved in chloroform was dried using nitrogen, resuspended to $7 \mathrm{mM}$ in the above kinase buffer and then sonicated for $30 \mathrm{~min}$ before use. Protein $1 \mu \mathrm{l}\left(1 \mathrm{mg} \mathrm{ml}^{-1}\right)$ was mixed with $7 \mu \mathrm{l}$ kinase buffer and $10 \mu \mathrm{l}$ PI micelles. The kinase reaction was initiated by adding $2 \mu \mathrm{l}$ ATP $(10 \mathrm{mM})$ and carried out at room temperature (RT). All reactions were repeated three times and were controlled with blank reactions that lacked PI. Reactions were stopped by addition of $20 \mu \mathrm{l}$ ADP-Glo reagent (Promega, USA). After 70 min incubation at RT, $40 \mu \mathrm{l}$ of Kinase Detection Reagent was added and the mixture was incubated for another $60 \mathrm{~min}$ at RT. The fluorescence signal of the mixture was recorded on a Luminescence Spectrometer which was set to a sensitivity of $30 \%$.

\section{Technique to determine the Crystal structure of PI4Kinases:}

Purified PI4KII $\alpha\left(\sim 30 \mathrm{mg} \mathrm{ml}^{-1}\right)$ is diluted with a buffer containing $25 \mathrm{mM}$ HEPES, $\mathrm{pH} 7.5,150 \mathrm{mM}$ $\mathrm{NaCl}, 1 \mathrm{mM}$ DTT and $20 \mathrm{mM} \mathrm{MgCl}$ to a final concentration of $4 \mathrm{mg} \mathrm{ml}^{-1}$ [18].The detergent hexaethylene glycol monooctyl ether was added to a final concentration of $20 \mathrm{mM}$. After incubation on ice for $40 \mathrm{~min}$, the protein solution was centrifuged at $15000 \mathrm{~g}$ for $30 \mathrm{~min}$. The pellet was discarded and the supernatant was collected for crystallization. Crystals of PI4KII $\alpha$ were grown by the hanging-drop vapour diffusion method at $293 \mathrm{~K}$ by mixing $1 \mu \mathrm{l}$ of protein solution with an equal volume of reservoir solution containing $0.1 \mathrm{M}$ citric acid, pH 5.8, $150 \mathrm{mM} \mathrm{NaCl}$ and 18\% PEG400. The crystal structure of the PI4K II alpha has been solved. The structure revealed an unusual kinase fold that could be divided between $\mathrm{N}$ - and $\mathrm{C}$ - lobes. The catalytically important ATP molecule is localized between the N- and C- lobes but surprisingly we found a second ATP molecule bound to a lateral pocket of the C-lobe. The adenine ring of the ATP is held inside the pocket mainly by hydrophobic interactions. We were able to show that the ATP is not the biological ligand for the lateral hydrophobic pocket but rather a suitable ligand present at or within the lipid bilayer is and the lateral pocket fine tunes the enzyme's activity in vivo.

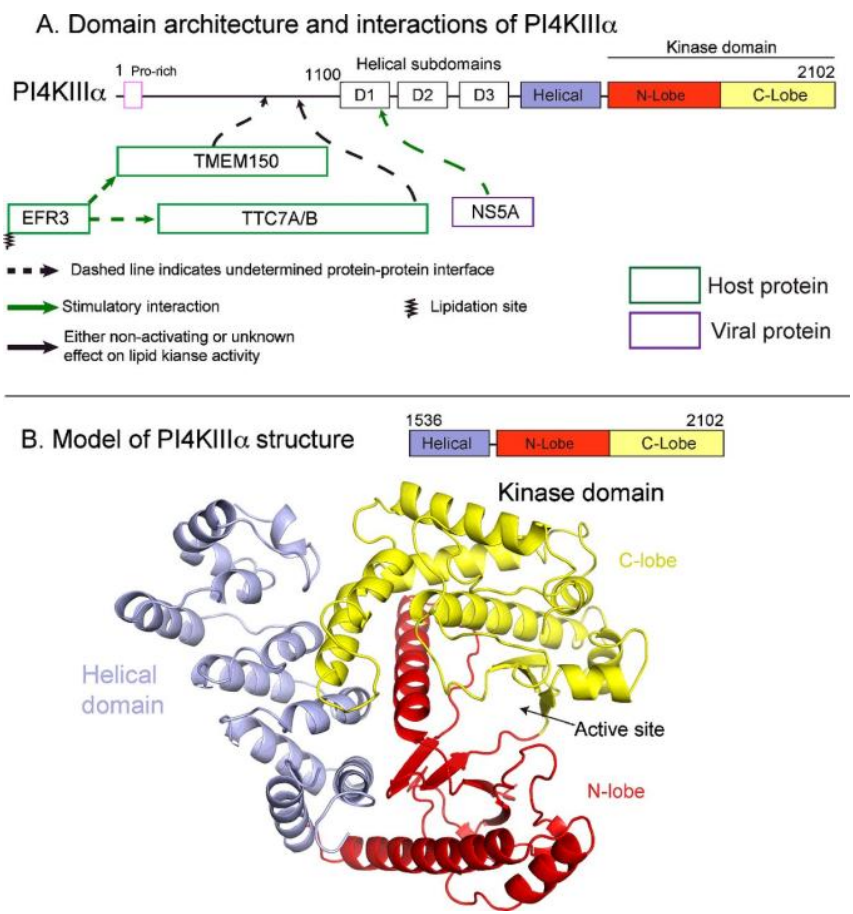

Fig.1: Structure of PI-4KIII ( Courtesy: Gillian L. Dornan, Jacob A. McPhail, John E. Burke, Biochemical Society Transactions Feb 09, 2016, 44 (1) 260-266 
Functional roles of PI4KII enzymes: It plays a important role in the development of a migratory phenotype in HCC but there is limited evidence for the direct involvement of mammalian PI-4KII isozymes in actin regulation. In contrast, several studies have described PI-4KII functions in post-TGN traffic through PI(4)P biosynthesis and direct binding to clathrin adaptors AP-1 and AP-3 [19], both pathways that contribute to endo-lysosomal traffic (Refer Fig.1). PI-4KII $\alpha$ controls LE traffic of the EGFR [20], endolysosomal traffic mediated by AP-3 and of lysosomal membrane proteins and hydrolases such as the enzyme $\beta$-glucocerebosidase [21].This explains the similar effects of PI-4KII $\alpha$ and PI-4KII $\beta$ depletion on EGFR degradation and LE traffic. Keeping in mind that most PI4KII $\alpha$ and a substantial portion of PI-4KII $\beta$ exist as membrane proteins in the cytoplasm owing to the palmitoylated cysteine residues, it appears inevitable that the nuclear PI4KII $\alpha$ and II $\beta$ also exist as membrane proteins in the nucleus. Moreover, in view of the fact that the several small Ins(1,4,5) $P_{3}$-sensitive nucleoplasmic $\mathrm{Ca}^{2+}$ store vesicles are the only known nucleoplasmic entity with membranes, the localization of PI4KII $\alpha$ and II $\beta$ in the nucleoplasmic vesicles is in accordance with their biochemical properties [22]. In addition to this nucleoplasmic $\mathrm{Ca}^{2+}$ store vesicles not only contain the high capacity, low affinity $\mathrm{Ca}^{2+}$ storage proteins chromogranin $\mathrm{B}$ and secretogranin II but also are loaded with the $\operatorname{Ins}(1,4,5) P_{3} \mathrm{R} / \mathrm{Ca}^{2+}$ channels [23].

Therapeutic potential of targeting the PI4-kinase enzyme: A further challenge in targeting the PI-4-kinases has emerged from more recent studies which have revealed differential cell and tissue dependencies on the PI 4-kinase isoforms that are not always absolutely predictable. Srivastava et al have shown that in T cell leukemia cells the PI-4 kinase associate with TCR- CD cells and this can be a potential for drug target and lead to curative prospects in blood cancers [24]. Another example is pertaining to the animal studies have shown that genetic knockdown of PI-4KIII $\alpha$ leads to severe changes in the gastrointestinal mucosal epithelium whereas loss of PI4KII $\alpha$ leads to selective loss of specific neuronal cell populations such as cerebellar Purkinje cells [25]. Therefore, it is not yet clear if inhibiting individual PI 4-kinase isoforms in a therapeutic setting represents a feasible strategy [26]. Imidazopyrazines demonstrate potent preventive, therapeutic, and transmission-blocking activity in rodent malaria models, are active against blood-stage field isolates of the major human pathogens $P$. falciparum and P. vivax, and inhibit liver-stage hypnozoites in the simian parasite P. cynomolgi. It has been shown that imidazopyrazines exert their effect through inhibitory interaction with the ATP-binding pocket of $\mathrm{PI}(4) \mathrm{K}$, altering the intracellular distribution of phosphatidylinositol-4-phosphate [27-28]. Collectively, these data define PI(4)K as a key Plasmodium vulnerability, opening up new avenues of target-based discovery to identify drugs with an ideal activity profile for the prevention, treatment and elimination of malaria. However, a more comprehensive knowledge of the roles of different PI 4-kinase permutations in modifying lipid metabolism, signalling and trafficking, may reveal which PI4P pathways to target in human diseases [29-31] . An excellent example of this is the Gaucher disease which is a lysosomal storage disorder caused by a defect in the degradation of glucosylceramide catalyzed by the lysosomal enzyme $\beta$-glucocerebrosidase (GBA). GBA reaches lysosomes via association with its receptor, lysosomal integral membrane protein type 2 (LIMP-2). It was found that distinct phosphatidylinositol 4-kinases (PI4Ks) play important roles at multiple steps in the trafficking pathway of the LIMP-2/GBA complex. Acute depletion of phosphatidylinositol 4-phosphate in the Golgi caused accumulation of LIMP-2 in this compartment, and PI4KIII $\beta$ was found to be responsible for controlling the exit of LIMP-2 from the Golgi. In contrast, depletion of PI4KII $\alpha$ blocked trafficking at a post-Golgi compartment, leading to accumulation of LIMP-2 in the endosomal vesicles. PI4KII $\alpha$ depletion also caused secretion of missorted GBA into the medium, which was attenuated by limiting LIMP-2/GBA exit from the Golgi by PI4KIII $\beta$ inhibitors [32]. These studies identified PI4KIII $\beta$ and PI4KII $\alpha$ as important regulators of lysosomal delivery of GBA, revealing a new element of control to sphingolipid homeostasis by phosphoinositides. The further goal is to get atomic insight in the PI4P biosynthesis that takes place at intracellular membranes of all eukaryotic organisms and to use the structural information to design specific inhibitors targeting PI-4Ks.

\section{Conclusions}

Future work to visualize and selectively deplete PI4P either in the ER or the Golgi apparatus may lead to better understanding of the roles of the pools of PI4P in each of these organelles that are important for biosynthetic trafficking. The Sac1 phosphatase cycles between the ER and Golgi apparatus and is a key regulator of PI4P in mammalian cells. While the localization of PI4P to the Golgi has been extensively reported, new tools to visualize PI4P pools in cells have revealed pools of this lipid associated with the PM and late endosomes/lysosomes. It will be interesting and informative to test whether these probes are capable of recognizing an ER-associated pool of PI4P in this cell system. Alternatively, further support for the necessity of PI4KIII $\alpha$ activity, as implicated by our pharmacological approach, could be provided with the use of siRNA to genetically silence this group of enzymes. 


\section{References}

[1]. Srivastava R., Ratheesh A., Rajiv K. Gude, R.K., Rao K.V.K ., Panda D and Subrahmanyam G. (2005) Resveratrol inhibits type II phosphatidylinositol 4-kinase: A key component in pathways of phosphoinositide turn over. Biochemical Pharmacology, 70, 1048-1055.

[2]. Balla A, Balla T. Phosphatidylinositol 4-kinases: old enzymes with emerging functions. Trends Cell Biol. 2006;16:351-61

[3]. Minogue S, Waugh MG. The phosphatidylinositol 4-kinases: don't call it a comeback. Subcell Biochem. 2012;58:1-24

[4]. De Matteis MA, Wilson C, D'Angelo G. Phosphatidylinsoitol-4-phosphate: the golgi and beyond. Bioessays. 2013;35:612-22.

[5]. Barylko B, Gerber SH, Binns EE, Grichine N, Khvotchev M, Sudhof TC,Joseph P. Albanesi .A novel family of phosphatidylinositol 4-kinases conserved from yeast to humans. J Biol Chem. 2001;276:7705-8.

[6]. Wong K, Meyers R, Cantley LC. Subcellular locations of phosphatidylinositol 4-kinase isoforms. J Biol Chem. 1997;272:13236-41

[7]. J. Li, Y. Lu, J. Zhang, H. Kang, Z. Qin, C. Chen.PI4KIIalpha is a novel regulator of tumor growth by its action on angiogenesis and HIF-1alpha regulation.Oncogene, 29 (2010), pp. 2550-2559

[8]. Altan-Bonnet N, Balla T. Phosphatidylinositol 4-kinases: hostages harnessed to build panviral replication platforms. Trends Biochem Sci. 2012;37:293-302

[9]. S. Ishikawa, H. Egami, T. Kurizaki, J. Akagi, Y. Tamori, N. Yoshida, et al.Identification of genes related to invasion and metastasis in pancreatic cancer by cDNA representational difference analysis.J Exp Clin Cancer Res, 22 (2003), pp. 299-306

[10]. V. Giroux, J. Iovanna, J.C. Dagorn. Probing the human kinome for kinases involved in pancreatic cancer cell survival and gemcitabine resistance.FASEB J, 20 (2006), pp. 1982-1991

[11]. D.A. Altomare, J.R. Testa.Perturbations of the AKT signaling pathway in human cancer.Oncogene, 24 (2005), pp. 7455-7464

[12]. K. Chu, S. Minogue, J. Hsuan, M. Waugh.Differential effects of the phosphatidylinositol 4-kinases, PI4KIIalpha and PI4KIIIbeta, on Akt activation and apoptosis.Cell Death Dis, 1 (2010), p. e106

[13]. Balla A, Kim YJ, Varnai P, Szentpetery Z, Knight Z, Shokat KM, et al. Maintenance of hormone-sensitive phosphoinositide pools in the plasma membrane requires phosphatidylinositol 4-kinase III $\alpha$ Mol Biol Cell. 2008;19:711-21

[14]. M. Jovic, M.J. Kean, Z. Szentpetery, G. Polevoy, A.C. Gingras, J.A. Brill, et al.Two phosphatidylinositol 4-kinases control lysosomal delivery of the Gaucher disease enzyme, beta-glucocerebrosidase.Mol Biol Cell, 23 (2012), pp. 1533-1545

[15]. Huh YH, Huh SK, Chu SY, Kweon HS, Yoo SH. Presence of a putative vesicular inositol 1,4,5-trisphosphate-sensitive nucleoplasmic $\mathrm{Ca}^{2+}$ store. Biochemistry 2006; 45:1362 - 73

[16]. Yoo SH, Chu SY, Kim KD, Huh YH. Presence of secretogranin II and high-capacity, low-affinity Ca2+ storage role in nucleoplasmic Ca2+ store vesicles. Biochemistry 2007; 46:14663 - 71

[17]. Mejdrova, I., Chalupská D, Kögler M, Šála M, Plačková P, Baumlová A, Hřebabecký H, Procházková E, Dejmek M, Guillon R, Strunin D, Weber J, Lee G ${ }^{1}$, Birkus G, Mertlíková-Kaiserová H, Boura E, Nencka R. Highly selective PI4K IIIbeta inhibitors and structural insight into their mode of action. J Med Chem, 2015, 14;58(9):3767-93.

[18]. Baumlova, A., Chalupska D, Róźycki B, Jovic M, Wisniewski E, Klima M, Dubankova A, Kloer DP, Nencka R, Balla T, Boura E. The crystal structure of the phosphatidylinositol 4-kinase IIalpha. EMBO Rep, 2014. 15(10): p. 1085-92.

[19]. Wang YJ, Wang J, Sun HQ, Martinez M, Sun YX, Macia E, Kirchhausen T., Albanesi J. P., Roth M. G., Yin H. L. Phosphatidylinositol 4 phosphate regulates targeting of clathrin adaptor AP-1 complexes to the Golgi. Cell. 2003;114:299-310

[20]. Nakatsu F, Baskin JM, Chung J, Tanner LB, Shui G, Lee SY, et al. PtdIns4P synthesis by PI4KIII $\alpha$ at the plasma membrane and its impact on plasma membrane identity. J Cell Biol. 2012;199:1003-16

[21]. Gillian L. Dornan, Jacob A. McPhail and John E. Burke. Type III phosphatidylinositol 4 kinases: structure,function, regulation, signalling and involvement in disease. Biochem. Soc. Trans. (2016) 44, 260-266

[22]. Jung G, Wang J, Wlodarski P, Barylko B, Binns DD, Shu H, Yin HL, Albanesi JP. Molecular determinants of activation and membrane targeting of phosphoinositol 4-kinase Ilbeta. Biochem J 2008; 409:501 - 9.

[23]. Balla A, Kim YJ, Varnai P, Szentpetery Z, Knight Z, Shokat KM, et al. Maintenance of hormone-sensitive phosphoinositide pools in the plasma membrane requires phosphatidylinositol 4-kinase III $\alpha$ Mol Biol Cell. 2008;19:711-21

[24]. Srivastava,R., Sinha R.K and Subrahmanyam G. (2006) Type II phosphatidylinositol 4-kinase $\beta$ associates with TCR-CD3 $\zeta$ chain in Jurkat cells. Molecular Immunology,43, 454-63.

[25]. J.P. Simons, R. Al-Shawi, S. Minogue, M.G. Waugh, C. Wiedemann, S. Evangelou, et al.Loss of phosphatidylinositol 4-kinase 2alpha activity causes late onset degeneration of spinal cord axonsProc Natl Acad Sci USA, 106 (2009), pp. 11535-11539

[26]. Bojjireddy, N., Botyanszki, J., Hammond, G., Creech, D., Peterson, R.,Kemp, D.C., Snead, M., Brown, R., Morrison, A., Wilson, S. et al. (2014)Pharmacological and genetic targeting of the PI4KA enzyme reveals its important role in maintaining plasma membrane phosphatidylinositol 4-phosphate and phosphatidylinositol 4,5-bisphosphate levels. J. Biol.Chem. 289, 6120-6132

[27]. Case W McNamara, Marcus CS Lee, Chek Shik Lim, Siau Hoi Lim, Jason Roland,Oliver Simon,Bryan KS Yeung,Arnab K Chatterjee,Susan L McCormack, Micah J Manary, Anne-Marie Zeeman, Koen J Dechering,TR Santha Kumar, Philipp P Henrich, Kerstin Gagaring, Maureen Ibanez, Nobutaka Kato, Kelli L Kuhen, Christoph Fischli, Advait Nagle, Matthias Rottmann, David M Plouffe,Badry Bursulaya, Stephan Meister, Lucia Rameh, Joerg Trappe, Dorothea Haasen, Martijn Timmerman, Robert W Sauerwein, Rossarin Suwanarusk, Bruce Russell,Laurent Renia, Francois Nosten, David C Tully,Clemens HM Kocken, Richard J Glynne, Christophe Bodenreider, David A Fidock,Thierry $\mathrm{T}$ Diagana,and Elizabeth A Winzeler.Targeting Plasmodium phosphatidylinositol 4-kinase to eliminate malaria. Nature. 2013 Dec 12; 504(7479): 248-253.

[28]. Bin Zou, Advait Nagle, Arnab K. Chatterjee, Seh Yong Leong, Liying Jocelyn Tan, Wei Lin Sandra Sim, Pranab Mishra, Prasuna Guntapalli, David C. Tully, Suresh B. Lakshminarayana, Chek Shik Lim, Yong Cheng Tan, Siti Nurdiana Abas, Christophe Bodenreider, Kelli L. Kuhen, Kerstin Gagaring, Rachel Borboa, Jonathan Chang, Chun Li, Thomas Hollenbeck, Tove Tuntland, Anne-Marie Zeeman, Clemens H. M. Kocken, Case McNamara, Nobutaka Kato, Elizabeth A. Winzeler, Bryan K. S. Yeung, Thierry T. Diagana, Paul W. Smith, and Jason Roland. Lead Optimization of Imidazopyrazines: A New Class of Antimalarial with Activity on Plasmodium Liver Stages. ACS Med. Chem. Lett., 2014, 5 (8), pp 947-950

[29]. F.H. Vaillancourt, M. Brault, L. Pilote, N. Uyttersprot, E.T. Gaillard, J.H. Stoltz, et al.Evaluation of Phosphatidylinositol-4-Kinase IIIalpha as a Hepatitis C Virus Drug Target. J Virol, 86 (2012), pp. 11595-11607

[30]. A.S. Guerreiro, S. Fattet, D.W. Kulesza, A. Atamer, A.N. Elsing, T. Shalaby, et al. A sensitized RNA interference screen identifies a novel role for the PI3K p110gamma isoform in medulloblastoma cell proliferation and chemoresistance.Mol Cancer Res, 9 (2011), pp. 925-935

[31]. Toth B, Balla A, Ma H, Knight ZA, Shokat KM, Balla T(2006) Phosphatidylinositol 4-kinase III $\beta$ regulates the transport of ceramide between the endoplasmic reticulum and Golgi. J Biol Chem. 281:36369-36377

[32]. Strahl, T., Hama, H., DeWald, D.B. and Thorner, J. (2005) Yeast phosphatidylinositol 4-kinase, Pik1, has essential roles at the Golgi and in the nucleus. J. Cell Biol. 171, 967-979 